Advanced Resource Planning as Decision Support Module to ERP

I. Van Nieuwenhuyse, L. De Boeck, M. Lambrecht and N. Vandaele

DEPARTMENT OF DECISION SCIENCES AND INFORMATION MANAGEMENT (KBI) 


\title{
Advanced Resource Planning as Decision Support Module to ERP
}

\author{
Inneke Van Nieuwenhuyse ${ }^{\mathrm{a}}$, Liesje De Boeck ${ }^{\mathrm{b}}$, Marc Lambrecht ${ }^{\mathrm{a}}$, Nico Vandaele ${ }^{\mathrm{ac}}$ \\ ${ }^{a}$ Research Center for Operations Management, Department of Decision Sciences and Information \\ Management, K.U.Leuven \\ inneke.vannieuwenhuyse@econ.kuleuven.be, marc.lambrecht@econ.kuleuven.be, \\ nico.vandaele@econ.kuleuven.be, \\ ${ }^{b}$ Center for Modelling and Simulation, HUBrussel, Affiliated with the Research Center for Operations \\ Management, Department of Decision Sciences and Information Management, K.U.Leuven \\ liesje.deboeck@hubrussel.be \\ NYO Alatus, a K.U.Leuven Spin-off \\ nico.vandaele@nyoalatus.com
}

\begin{abstract}
In this paper, we show that the planning and decision-support capabilities of the MPC (Manufacturing Planning and Control) system, which forms the core of any ERP (Enterprise Resource Planning) package, may be substantively enhanced by including a Decision Support Module (DSM) as an add-on at the midterm planning level. This DSM, called Advanced Resource Planning (ARP), serves as parameter setting process as well as tool for improving the structure of the ERP system itself. The ultimate goal of the DSM is to yield realistic information both for scheduling, sales and marketing, strategic and operational decision making and suppliers and customers.
\end{abstract}

\section{Introduction}

The emergence and widespread adoption of ERP systems undoubtedly constitutes one of the most pervasive changes in the business environment over the past decades. According to Vollmann et al. (2005), the term ERP can be interpreted in two ways.

1. From the point of view of the IT community, the emphasis is on integration: an ERP system is a software tool enabling to integrate the different application programs (HR, finance, sales, marketing, production planning,...) in a company, by efficiently tracking all transactions in real time and sharing them across all functions through a common database. In the light of today's market environment, one can view the integration aspect of ERP as a prerequisite for further improvement. Organizations are increasingly aware that the next step in increasing profit and market share consists in engaging in effective supply chain management (Simchi-Levi et al., 2000). Indeed, the emergence of the specialized company has led to a surge in the number of partners contained in a single chain. Moreover, these partners tend to be spread around the globe. The success and widespread implementation of ERP systems has laid the groundwork for further integration across the entire supply chain.

2. On the other hand, managers tend to emphasize the planning aspect: an ERP system should be able to support decisions regarding the planning and execution of the business. Indeed, Berchet and Habchi (2005) point out that production planning is one of the most important modules supporting ERP systems. Botta-Genoulaz and Millet (2005) also provide evidence on this statement by showing through surveys that production planning and control (PPC) belongs to the most frequently implemented ERP modules. However, it is well known that the strength of ERP is not situated in the planning area (Stadtler, 2005). That is largely because ERP nowadays still exhibits many weaknesses of MRP (from which it originates (see e.g. Miltenburg, 2001; Pairat and Jungthirapanich, 2005; Berchet and Habchi, 2005; Jacobs and Weston Jr., 2006)), as its production modules are made up of standard Material Requirements Planning (MRP) logic (see e.g. Silver et al., 1998): except from the old logic executed much faster and in real time, little has 
changed since the 70's. Along the same lines, Stevenson et al. (2005) indicate that the core planning and control assumptions underpinning ERP have less rapidly developed than the new software capabilities within ERP systems.

So instead of concentrating on the decision-support capabilities for the planning and execution, ERP systems have tended to focus on their integration aspect for which they are primarily known (Holsapple and Sena, 2003). And this in spite of the decision-making support being considered fairly important as a reason for adopting ERP (Holsapple and Sena, 2005). From this, it is clear that additional decisionsupport in the planning area of ERP is necessary. This paper aids in bridging this gap by providing an Advanced Resource Planning (ARP) module as Decision Support Module (DSM) to ERP. Indeed, it will become clear in what follows that the module helps in setting more realistic PPC parameters (which is one of the stumbling blocks of PPC as reported by Wiendahl et al. (2005)), dynamically adjusting the parameters through some sort of Parameter Review Process and improving the structure of the ERP system itself. The development of the ARP module is also in line with the idea advocated by Jacobs and Weston (2007) that the academic community should play a much more active role in the development of intelligent software logic that can be used by real companies with respect to ERP.

The paper is organized as follows. In the next section, we zoom in on the motivation for including ARP as DSM in ERP. In Section 3, we present some fundamental insights on the dynamic behavior of manufacturing systems, and their implications for MPC modeling and decision support. In Section 4, we present the framework for the integration of ERP and ARP. Section 5 highlights the decision-support benefits of ARP and Section 6 outlines the conclusions, and summarizes the limitations and benefits of our approach.

\section{Motivation for including Advanced Resource Planning as Decision Support Module for ERP}

ERP systems evolved out of traditional Manufacturing Planning and Control (MPC) systems. As shown in Figure 1 (adapted from Vollmann et al. (2005)), the MPC system still constitutes the core of any ERP system. It reflects the hierarchy of planning, with Sales and Operations Planning (S\&OP) on the long term level, Master Production Scheduling (MPS) and Material Requirements Planning (MRP) at the midterm level, and Shop Floor Control (SFC) and Supplier Systems at the short term level.

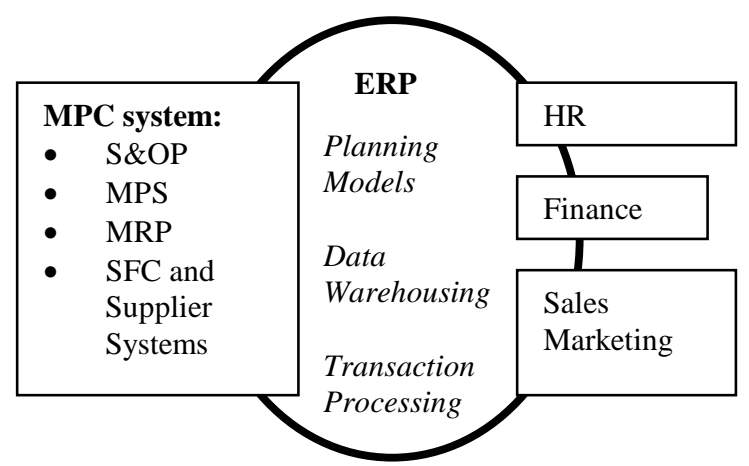

Figure 1. The scope of the ERP system

The feasibility of the operational improvements that managers expect from an ERP implementation (such as lead time reductions, realistic capacity planning, improved on-time delivery, alignment of sales to planning) largely depends upon the effectiveness of the embedded MPC system. 
In this paper, we show that the capabilities of the MPC system may be substantively enhanced by explicitly recognizing the variability (uncertainty) and the limited production capacity of manufacturing systems. Variability is inherent to real-life systems. Recent research in the Operations Management and Operations Research fields (e.g., see Suri et al., 1993; Buzacott and Shantikumar, 1993; Lambrecht et al., 1998; Hopp and Spearman, 2008) has shown that variability has a substantive impact on the dynamic behavior of a manufacturing system, and hence on operational parameters such as production lead times and obtained customer service levels. Though ERP requires these parameters as input to the MPC planning module (e.g., for lead time offsetting in MRP calculations), current systems are not yet equipped to take this impact into account: the input parameters for the MPC module are mostly estimated (or simply fixed) in an ad hoc fashion. This often leads to unrealistic parameter settings, which undermine the effectiveness of the MPC system. This is confirmed by a survey of Botta-Genoulaz and Millet (2005) showing that with respect to lead time control through ERP systems, the realized control versus the expected control was only around half. Remark that also Koh and Saad (2003) relate the underperformance of ERP to the uncertainty in the type of environments they operate in. They report on reactive instead of proactive reactions to uncertainty using ERP because of a lack of attention for the underlying causes of uncertainty. In the following sections, we will point out that this dynamic behavior is an inescapable consequence of system variability (or as Selçuk at al. (2006) put it: the stochastic nature of the manufacturing system is a significant characteristic of dynamic environments). Good estimates of this behavior are thus a prerequisite for a successful MPC system. Traditional transaction oriented ERP systems not only ignore the importance of resource allocation decisions under uncertainty (variability), but also largely ignore the impact of limited production capacity. Indeed, on the midterm planning level, resources are typically limited in capacity: though capacity may be increased (or decreased) to a certain extent, the amount of capacity available is not infinitely flexible. Furthermore, manufacturing systems nowadays operate in an increasingly complex environment. The surge in product customization and the emphasis on time-based competition complicate planning efforts, and require today's businesses to be increasingly responsive. In terms of the MPC system, this translates into the need for planning tools that not only yield realistic information on planning parameters, but are also able to generate this desired output in "no time".

When it comes to relating decision-support benefits and ERP in general, Holsapple and Sena (2005) note that these benefits have not been identified and discussed with ERP systems. Up to now, ERP systems are still known primarily for their transactional characteristics rather than for their decisionsupport characteristics (Holsapple and Sena, 2003). Nevertheless, ERP is provided to aid decisionmaking (Stevenson et al., 2005). Indeed, the primary objective of the ARP module is to obtain a more accurate and realistic view on the company's key operational indicators (such as effective capacity, inventory and lead times) and how they are impacted by managerial decisions such that decision-making is supported in a more accurate way. As such, it provides knowledge for other functional areas and involved parties as suppliers and customers (see infra) or putting it in terms of Desanctis and Gallupe (1987): reducing of uncertainty or noise during decision-making" (see also Holsapple and Sena, 2003) and it helps to improve the reliability of decision processes or outcomes.

\section{Basic system dynamics}

In this section, we present some fundamental insights on the dynamic behavior of a stochastic system. Figure 2 shows the basic factors which determine the performance of a manufacturing system, such as lead time behavior and customer service levels (on-time delivery performance).

The major determinants of lead time behavior are effective utilization of the manufacturing system (see Hopp and Spearman, 2008) and variability. Utilization is an average concept, which results from the 
confrontation of demand and supply. The demand side represents the customers, who place orders and consequently put a load on the manufacturing system. This load depends on both the quantity and timing of all incoming customer orders. On the other hand, the supply side represents the capacity, i.e., the resources (which may consist of machines, transportation resources, personnel etc.) of which the manufacturer disposes in order to fulfill customer demands. Next to utilization, we have variability as a second determinant of system performance. As noted in the previous section, variability is inherent to real-life systems, both at the demand side and at the supply side. Different customer orders all consisting of different product types in different quantities arriving at different times, cause demand variability. At the supply side, resources are not always available for production: planned and unplanned outages (such as shift patterns, preventive maintenance or equipment failures) are sources of variability and lead to a decrease in the capacity of the resources at hand. The same accounts for machine setup times. Note that by e.g. these capacity decreases, these outages influence utilization. Utilization affects variability since it is an important factor in the propagation of variability to downstream stations in a manufacturing line (see the linking equation in Hopp and Spearman (2008)).

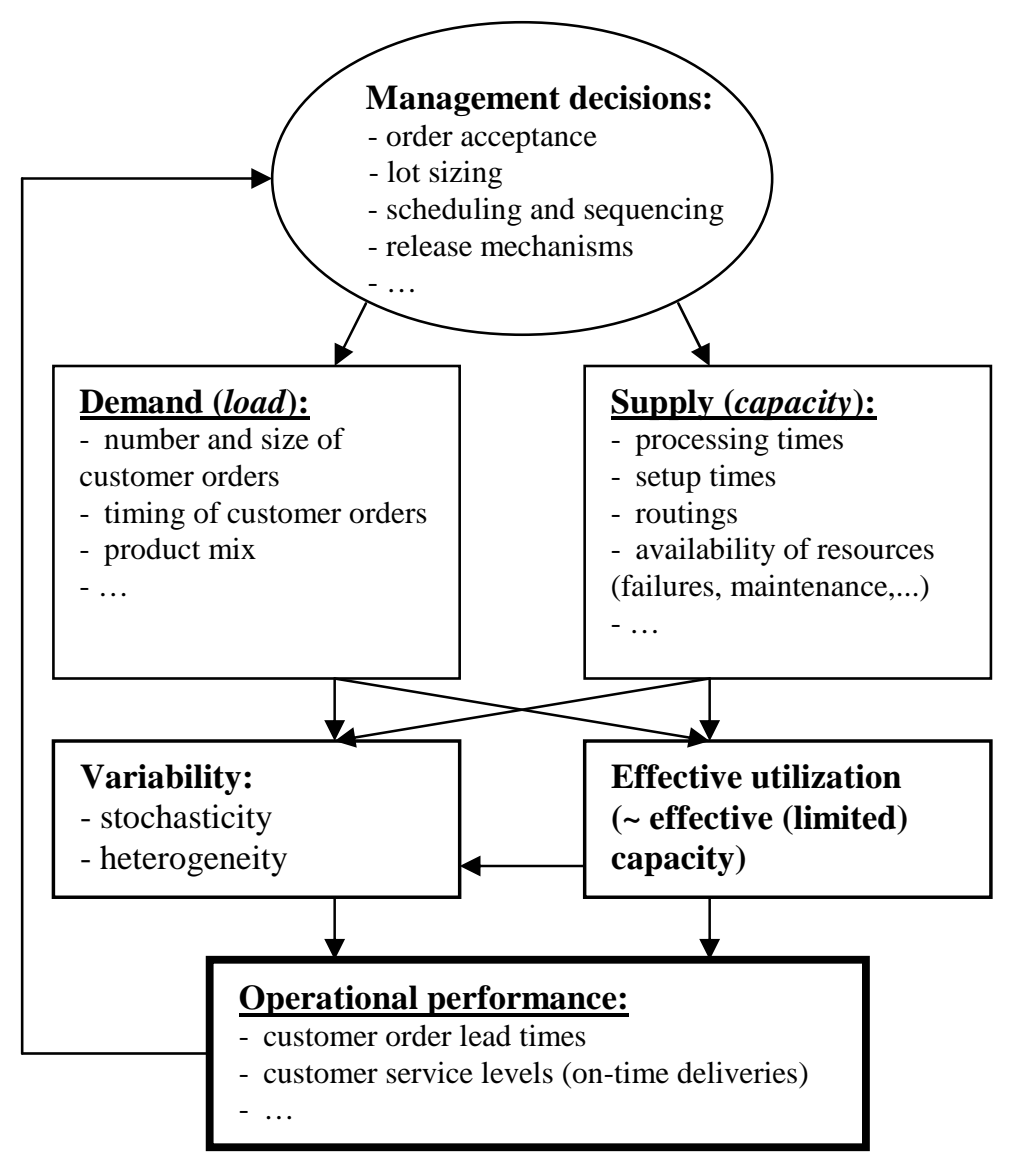

Figure 2. Determinants of operational performance of a manufacturing system

The resulting capacity, taking into account all "outages" (e.g., machine breakdowns, quality problems, material shortages, setup times) is called the effective capacity. The division of the load put on the system by the effective capacity results in the effective utilization. As the effective capacity is finite, the load that is put on the system will result in competition for resources. In a system that is subject to variability, this competition causes congestion, and average lead times will increase. Congestion occurs 
even when the ratio between load and effective capacity is such that the system remains feasible (effective utilization below 100\%). This relationship between effective utilization and average lead time performance is highly nonlinear, as depicted in Figure 3 (see Hopp and Spearman, 2008). Moreover, variability acts as an amplifier on congestion: the higher the variability in the system, the more congestion will occur for a given effective utilization. At this point, we arrive at one of the decision support capabilities of the ARP-module (see also Section 5), i.e. strategic and operational decision making. Management can intervene (parameter tuning,...) to influence operational performance. However, management decisions (see Figure 2) may impact system performance in both a favorable or unfavorable way. Decisions regarding order acceptance, lot sizing (including both process lot sizing and transfer lot sizing), scheduling and sequencing and order release may influence demand side and supply side parameters and therefore also the effective utilization of the system as well as the inherent system variability (see e.g. Karmarkar et al., 1985a; Karmarkar et al., 1985b; Kekre, 1987; Lambrecht et al., 1996; Van Nieuwenhuyse et al., 2000; Dupon et al., 2002). As example of these management decisions, we now zoom in on the lot sizing decision. The relationship between lot sizing and lead time has been the most thoroughly studied up to date. It is now widely accepted that the relationship between process lot size and average lead time is convex (e.g., Karmarkar, 1987 through Kekre, 1987; Lambrecht et al., 1996; Lambrecht et al., 1998; Vandaele et al., 2003; Van Nieuwenhuyse et al., 2000; Van Nieuwenhuyse et al., 2007), implying that there exists an optimal lot size minimizing average lead time. This insight has led to the development of optimization procedures, which make use of the convexity property to determine the "optimal" process lot sizes for a given objective function in multi-product multi-machine settings (see e.g. Lambrecht et al., 1998; Vandaele et al., 2003; Van Nieuwenhuyse et al., 2007 for applications of these procedures). In contemporary planning systems, lot sizing decisions are mostly taken in an ad hoc fashion, determined by means of a cost optimization procedure (such as EOQ or Wagner Whitin based procedures), or determined by a scheduler (mostly based on a heuristic). Regardless of the approach used, the impact on effective utilization and lead time behavior is currently ignored. This may lead to dangerous situations, particularly in environments with long setup times. In these settings, applying lot sizes that are too small may cause the effective utilization to rise beyond $100 \%$, de facto leading to an infeasible system. For that reason, the incorporation of an optimization procedure into the planning system at an aggregate (midterm) level would undoubtedly constitute a major advance in the management decision-support capabilities of the MPC system.

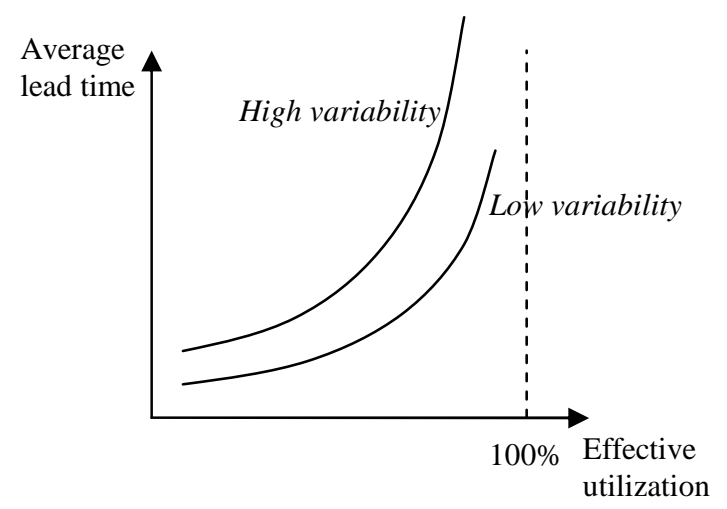

Figure 3. Relationship between effective utilization and average lead time

The effect demonstrated in Figure 3 may be quantified by queueing expressions (see e.g. Hopp and Spearman, 2008). This figure clearly reveals that the average lead time increases in a highly non-linear fashion for increasing values of the effective utilization. On top, the figure demonstrates the corrupting 
influence of variability: higher variability leads to more congestion. Any real-life manufacturing system is subject to variability, and will hence inescapably behave as depicted in Figure 3. Indeed, at the demand side, the timing and quantity of customer orders are typically stochastic. At the supply side, processing and setup times are usually stochastic too, and dependent upon the product type in case of a heterogeneous product mix. Unavailability of the resources adds further variability to the supply side.

From Figure 3, we may draw a number of conclusions that are a prerequisite for good manufacturing planning practice. Firstly, it is fundamental to recognize the importance of both limited effective capacity and variability when developing plans at the midterm level, as these are the drivers of lead time behavior. Current MPC systems however contain no tools to evaluate effective capacity or variability, let alone to adequately model the resulting dynamics. Secondly, it turns out that high utilization and low average lead times are incompatible in real life systems. Planning your system at full capacity (effective utilization of $100 \%$ ) is infeasible without further intervention: this would cause the average lead time to soar to infinity (Hopp and Spearman, 2008). Even an effective utilization close to $100 \%$ causes the manufacturing system to be out of breath, leading to unacceptably long lead times due to the long waiting times, and moreover to vast amounts of money tied up in working capital, especially inventories. In traditional ERP systems, lead-time is considered as fixed regardless of the level of effective utilization; consequently, lead times are often dramatically underestimated. The fundamental trade-off which is shown in Figure 3 should be taken into account when devising material and capacity plans: in current MPC systems, no link is made between the two performance measures, leading to plans which often assume both high capacity utilization (often in view of "efficient" resource utilization) and attractive (target or "wishful thinking") lead times, and hence are inherently unrealistic. The consequences of this approach will be primarily noticeable on the short-term (SFC) planning level: unrealistic targets at the midterm level unnecessarily complicate the scheduling effort, and lead to nervousness and increased fire-fighting behavior on the shop floor. Moreover, these efforts will likely be to no avail: smart scheduling is rarely able to correct for fundamental errors made at the midterm planning level. Only realistic lead time information allows a manager to determine acceptable release and due dates, i.e. providing the right time windows for scheduling and sequencing on the operation level (Vandaele and De Boeck, 2003; Lambrecht et al., 1998).

While the discussion so far has focused on average lead time behavior, it is primordial to recognize that, as the manufacturing system is subject to variability, the resulting lead time (of customer orders, process lots, etc.) will be a stochastic variable too. The factors shown in Figure 2 (effective utilization, variability, and management decisions) will also impact the variability of the lead time, and even the entire lead time probability distribution. Research (Dupon et al., 2002; Vandaele and De Boeck, 2003; Vandaele and Lambrecht, 2003) has revealed that the lead time distribution is typically positively skewed with a heavy tail. Insight into the lead time distribution is primordial in two respects regarding improving the reliability of decision processes.

Firstly, it impacts issues regarding customer service levels. Indeed, the percentiles of the lead time distribution determine the customer service level that the company will obtain for a given agreed lead time. Equivalently, insight into the distribution supports managers in quoting realistic lead times to their customers, in view of obtaining a target customer service level. Secondly, it promotes realistic lead time offsetting in the Material Requirements Planning (MRP) context. In order to ensure due date compliance and robust schedules in production, the lead times used in MRP should incorporate safety time. This safety time can be quantified from the lead time percentiles, and determines a realistic time window for order release and order completion. Hence, an adequate estimate of the lead time distribution is essential for a stable and smoothly running MPC system.

Apart from the ARP module as parameter setting process, it is also a tool that can be used to improve the structure of ERP systems. As such, the safety time issue also has implications on the practical design 
of ERP systems. E.g., it has been shown that it is advisable to limit the number of levels in the Bill-OfMaterial, for the simple reason that detailed allocated safety time is inferior to pooled safety time (Vandaele and Lambrecht, 2003). The same holds for the number of tiers in a supply chain (Welborn, 2008). In other words, the design of the ERP system itself will impact lead time performance.

\section{ERP and ARP: framework}

The backbone of the ARP system is an open queueing network model, which models the system on an aggregate level. Though this approach is approximative and does not enable great detail, we are confident that it is sufficiently precise for supporting midterm planning. Real-life applications of this open queueing network model speak in favor of this approximative model (Lambrecht et al., 1998; Vandaele et al., 2002; Vandaele and Lambrecht, 2002; Vandaele et al., 2000; Vandaele et al., 2003; Claerhout et al., 2005). Moreover, queueing network models require short run times, as opposed to (for example) discreteevent simulation models (Suri et al., 1995). As mentioned in Section 2, this aspect is crucial in order to support management decision making in today's complex environment. Another important advantage over discrete-event simulation is the fact that queueing network models are largely generic, making them particularly appropriate for what-if analyses and widespread use in different industry settings. As the literature on open queueing networks is vast, we have chosen not to go into details regarding the technical expressions in this paper; the interested reader can find further information in e.g. Suri and de Treville (1992), Suri et al. (1993), Buzacott and Shantikumar (1993), and Lambrecht et al. (1998). Instead, we will focus on the interface of ERP and ARP, i.e., which input parameters are needed for the ARP module, and which output parameters are fed back into the MPC system.

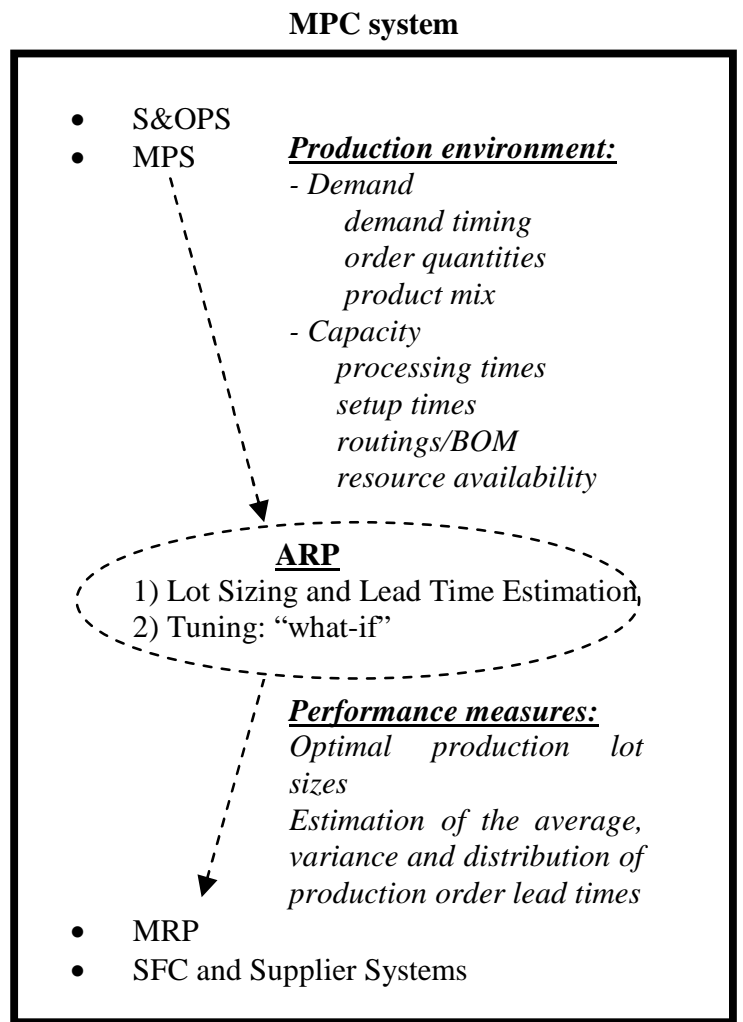

Figure 4. Framework for integrating the ARP module into ERP 
As shown in Figure 4, ARP uses input parameters which are available on the level of S\&OP and the resulting MPS. As stated in Vollmann et al. (2005), the MPS constitutes the anticipated build schedule for the company: it specifies the quantities of end products to be completed during the next planning period, with a required completion time. The MPS is usually constructed on a sufficiently long planning horizon (usually several months), and is mostly updated every couple of weeks. From the MPS (and S\&OP), we obtain information about the demand side for the upcoming planning period (i.e., which products need to be produced, what is the timing of the demand, and what are the quantities). This information enables us to quantify the load, as well as the variability inherently present in the demand. On the other hand, the information about the supply side (such as routings of the products that must be produced, setup and processing times on the different workcenters, failure behavior of resources, planned outages) is usually available from standard company records (bill of material, routing files, resource files, resource availability lists) which are contained in the ERP database. This information is crucial for the ARP module, as it determines effective capacity.

The ARP module translates the characteristics of the production environment for the upcoming planning period into the following output estimates: optimal production lot sizes, average and variance of the order lead time, average and variance of the waiting times in queue in front of the different workcenters, and lead time distribution. This information may be retrieved for every end item and/or component. Two phases may be distinguished in the ARP procedure (see also Claerhout et al., 2005; Lambrecht et al., 1998). During the lot sizing and lead time estimation phase, the manufacturing system is modelled as an aggregate queueing network in which all parameters are functions of the manufacturing lot sizes. By applying an optimization routine to this network, an optimal manufacturing lot size is obtained for every product type, along with estimates of the corresponding performance measures (average, variance and distribution of production order lead times and waiting times in queue). Next, the tuning phase enables management to adjust the capacity or demand structure through what-if analyses (by using overtime, implementing a capacity expansion, off-loading heavily loaded resources, or considering alternative routings) if the performance measures are considered unacceptable or if the proposed order portfolio leads to infeasibilities (e.g., resources which are used beyond effective capacity). Once the tuning phase is finished and management is satisfied with the estimated performance measures, the output is communicated to the next level in the MPC hierarchy, i.e. MRP, and further down to the level of SFC.

We may conclude that the integration of an ARP module into the current ERP systems may be done in a relatively straightforward way, as most of the required input data may be retrieved from ERP records. Hence, the ARP add-on can be merely considered as an intermediate calculation and optimization engine, necessary to enable robust planning and scheduling at the lower levels of the MPC system. The challenge, in our opinion, lies in ensuring that the information flow is managed in such a way that the ARP system delivers its full decision-support benefits (see Section 5). We think here for example of the timely dissemination of lead time related information to the sales department, in view of quoting delivery lead times. We would also like to stress the need for updating the relevant information at the start of every planning period.

\section{Decision-support benefits of ARP}

From the previous discussion, it is evident that the decision-support capabilities of an ERP system can be strongly enhanced by integrating an add-on ARP module, which enables to adequately quantify the dynamic behavior of the manufacturing system at hand. The ARP module should be seen as a DSM 
situated at the midterm (material and capacity planning) level, as shown in Figure 4. The decisionsupport benefits of this ERP add-on will be perceptible in numerous functional areas of the company:

(1) Scheduling (and execution): As the midterm plans devised by the MPC system will be based on more realistic information, it will be easier for the schedulers to devise short-term plans which fit within the anticipated time windows (Lambrecht et al., 1998). This entails both operational and financial advantages. As the effectiveness of the schedule improves, the people responsible for executing the schedule have little incentive to deviate from it, and hence schedule stability is improved. Moreover, material and components will be launched as needed, preventing shortages or excess of work-in-process on the shop floor. In this way, the company avoids unnecessary investments in working capital.

(2) Sales and marketing: for companies working in a make-to-order (MTO) environment, it is vital that the lead time quoted to the customers is reliable. Note that due to the increased demand for specialized products, this MTO sector is increasing in importance (Stevenson et al., 2005). The functionality of the ARP module allows to dynamically adjusting lead time quotations to changes in demand or shop floor conditions, such that the sales department's promises are in sync with the manufacturing department's capabilities and due date performance is secured. This is in line with Stevenson et al. (2005) who indicate that currently, ERP does not tackle the key characteristics of the MTO-industry such as the importance of accurate and competitive delivery date quotations at the customer inquiry stage.

(3) Strategic and operational decision making: the ARP module allows managers to fine-tune both strategic decisions (such as capacity investments, outsourcing decisions) and operational decisions (such as lot sizing) in view of the operational targets they want to achieve (e.g., lead time reduction). As such, the ARP module not only provides a tool for analysis, but also a lever for operational improvement. Although planning and scheduling models are frequently treated independently (Kreipl and Pinedo, 2004), we consider them linked with each other in line with the hierarchical production planning (HPP) philosophy (Hax and Meal, 1975). As a result, the decisions made at the higher planning levels impose constraints for the shorter planning levels.

Apart from these benefits, the ARP add-on brings advantages for the company's customers and suppliers too. It is self-evident that customers draw benefit from realistic lead time quotations. As the timing and quantity of purchase orders are generated by the MRP system based on the company's own build schedule, a stable schedule at the level of the company will translate into a more reliable order pattern (less expediting or de-expediting, fewer quantity changes, fewer rush orders,...) perceived by the supplier. In this way, the improved transparency and data accuracy provided by the ARP implementation improves supply chain effectiveness, and helps to mitigate one of the primary sources of the bullwhip effect.

The main goal of any ERP system consists in enhancing transparency, knowledge and information management for the company and its customers (clients and suppliers). In this respect, the importance of the add-on ARP module in providing realistic (i.e. reliable) information can hardly be understated.

\section{Conclusion}

In this paper, we have proposed the development of an Advanced Resource Planning module, as an add-on Decision Support Module to current ERP systems. This ARP module enables the planner to capture the relationship between variability and uncertainty on the one hand and capacity utilization, inventory (lead time) and customer service on the other hand. The approach is based on queueing approximations.

The decision-support characteristic related to this ARP deals with improving the reliability of decision processes in terms of providing (accurate) knowledge for other functional areas and involved parties such as suppliers and customers. In a nutshell, we can state that the ARP module provides support for 
management decision making at the midterm planning level. It allows for optimization of manufacturing lot sizes, and fine-tuning of the capacity and/or demand structure in view of obtaining target operational performance. As the approach is based on analytical models (instead of, for instance, simulation), the run time for these what-if analyses is typically very short, offering managers the timely decision-support needed in today's complex and ever-changing environment.

The output of the ARP module consists of realistic estimates of key planning parameters, such as expected production lead times and estimates of required safety times. These estimates offer useful information for different departments of the company. Firstly, they offer critical information to the schedulers, by determining realistic time windows for scheduling and sequencing, and setting reliable production order release dates. The ARP module stimulates the pro-active attitude of planners: as a result, nervousness and fire-fighting behavior on the shop floor are avoided. The estimates also offer the necessary information for reliable lead time quotations by the sales department.

One limitation of the approach is that it only offers estimates of the required parameters. As the ARP engine consists of a queueing model, the resulting output is approximative. This drawback is, however, hard to avoid. In order to capture the complexity of real-life manufacturing systems, queueing modelers have no choice but to resort to approximations. While simulation may offer greater precision than the queueing approach, we believe it is ill-suited for the intended purposes, as the resulting models are not generic and require long run times.

\section{References}

V. Botta-Genoulaz and P.A. Millet, A classification for better use of ERP systems, Computers in Industry 56 (2005) 573-587.

C. Berchet and G. Habchi, The implementation and deployment of an ERP system: an industrial case study, Computers in Industry 56 (2005) 588-605.

J.A. Buzacott and J.G. Shantikumar, Stochastic Models of Manufacturing Systems, (Prentice Hall, 1993).

D. Claerhout, N. Vandaele and I. Van Nieuwenhuyse, E-POLCA to control multi-product multimachine job shops, University of Antwerp Research paper 2005-007 (2005).

G. Desanctis and R.B. Gallupe, A foundation for the study of group decision support systems, Management Science 33 (1987) 589-609.

A. Dupon, I. Van Nieuwenhuyse and N. Vandaele, The impact of sequence changes on product lead time, Robotics and Computer-Integrated Manufacturing 18 (2002) 327-333.

A.C. Hax and H.C. Meal, Hierarchical integration of production planning and scheduling, Studies in Management Science 1 (1975) 53-69.

C.W. Holsapple and M.P. Sena, The decision-support characteristics of ERP systems, International Journal of Human-Computer Interaction 16 (2003) 101-123.

C.W. Holsapple and M.P. Sena, ERP plans and decision-support benefits, Decision Support Systems 38 (2005) 575-590.

W. Hopp and M. Spearman, Factory Physics, (Irwin, Signapore, 2008).

F.R. Jacobs and F.C. Weston Jr., Enterprise resource planning (ERP) - A brief history, Journal of Operations Management 25 (2007) 357-363.

U. Karmarkar, Lot sizes, lead times, and in-process inventories, Management Science 33 (1987) 409423.

U. Karmarkar, S. Kekre and S. Freeman, Lot sizing and lead time performance in a manufacturing cell, Interfaces 15 (1985a) 1-9.

U. Karmarkar, S. Kekre and S. Kekre, Lot sizing in multi-item multi-machine job shops, IIE Transactions 17 (1985b) 290-298. 
S. Kekre, Performance of a manufacturing cell with increased product mix, IIE Transactions 19 (1987) 329-339.

S.C.L. Koh and S.M. Saad, A holistic approach to diagnose uncertainty in ERP-controlled manufacturing shop floor, Production Planning \& Control 14 (2003) 273-289.

S. Kreipl and M. Pinedo, Planning and scheduling in supply chains: an overview of issues in practice, Production and Operations Management 13 (2004) 77-92.

M.R. Lambrecht, S. Chen and N.J. Vandaele, A lot sizing model with queueing delays. The issue of safety time, European Journal of Operational Research 89 (1996) 269-276.

M.R. Lambrecht, P.L. Ivens and N.J. Vandaele, ACLIPS: A capacity and lead time integrated procedure for scheduling, Management Science 44 (1998) 1548-1561.

J. Miltenburg, Computational complexity of algorithms for MRP and JIT production planning problems in enterprise resource planning systems, Production Planning \& Control 12 (2001) 198-209.

R. Pairat and C. Jungthirapanich, A chronological review of ERP research: an analysis of ERP inception, evolution, and direction, Proceedings of the IEEE International Engineering Management Conference 1 (2005) 288-292.

B. Selçuk, J.C. Fransoo and A.G. de Kok, The effect of updating lead times on performance of hierarchical planning systems, International Journal of Production Economics 104 (2006) 427-440.

E.A. Silver, D.F. Pyke and R. Peterson, Inventory Management and Production Planning and Scheduling (Wiley, 1998).

D. Simchi-Levi, P. Kaminsky and E. Simchi-Levi, Designing and Managing the Supply Chain (McGraw-Hill, 2000).

H. Stadtler, Supply chain management and advanced planning-basics, overview and challenges, European Journal of Operational Research 163 (2005) 575-588.

M. Stevenson, L.C. Hendry and B.G. Kingsman, A review of production planning and control: the applicability of key concepts to the make-to-order industry, International Journal of Production Research 43 (2005) 869-898.

R. Suri and S. de Treville, Rapid Modelling: the use of queueing models to support time-based competitive manufacturing, in: G. Fandel, T. Gulledge and A. Jones, Eds., Operations Research in Production Planning and Control (Springer-Verlag, New York, 1992).

R. Suri, G.W.W. Diehl, S. de Treville and M.J. Tomsicek, From CAN-Q to MPX: Evolution of queueing software for manufacturing, Interfaces 25 (1995) 128-150.

R. Suri, J.L. Sanders and M. Kamath, Performance evaluation of production networks, in: S.C. Graves, A.H.G. Rinnooy Kan, P.H. Zipkin, Eds., Logistics of Production and Inventory, (Elsevier Science Publishers, Amsterdam, 1993).

N. Vandaele and L. De Boeck, Advanced Resource Planning, Robotics and Computer Integrated Manufacturing 19 (2003) 210-218.

N. Vandaele, L. De Boeck and D. Callewier, 2002, An open queueing network for lead time analysis, IIE Transactions 32 (2002) 1-9.

N. Vandaele and M. Lambrecht, Planning and scheduling in an assemble-to-order environment: Spicer Off-Highway products division, in: J. Song and D. Yao, Eds., Supply Chain Structures: Coordination, Information and Optimisation, (Kluwer, 2002).

N.J. Vandaele and M.R. Lambrecht, Reflections on the use of stochastic manufacturing models for planning decisions, in: J.G. Shanthikumar, D.D. Yao and W.H.M. Zijm, Eds., Stochastic Modeling and Optimisation of Manufacturing Systems and Supply Chains, (Kluwer Academic Publishers, Boston 2003).

N.J. Vandaele, M.R. Lambrecht, N. De Schuyter and R. Cremmery, Spicer Off-Highway Products Division-Brugge improves its lead time and scheduling performance, Interfaces 30 (2000) 83-95. 
N. Vandaele, I. Van Nieuwenhuyse and S. Cupers, Optimal grouping for a nuclear magnetic resonance scanner, European Journal of Operational Research 151 (2003) 181-192.

I. Van Nieuwenhuyse, N.J. Vandaele and V. Dossogne, Better understanding lead times in terms of transfer batching and process batching, in: Information Technology and Operations Management: Relationships and Synergies, (Tata Mc-Graw Hill, New Delhi, 2000).

I. Van Nieuwenhuyse, N. Vandaele, K. Rajaram and U. Karmarkar, Buffer sizing in multi-product multi-reactor batch processes: impact of allocation and campaign sizing policies, European Journal of Operational Research 179 (2007) 424-443.

T.E. Vollmann, L.B. Berry, D.C. Whybark and F.R. Jacobs, Manufacturing Planning and Control Systems for Supply Chain Management (McGraw Hill, New York, 2005).

C. Welborn, Strengthening supply chains, OR/MS Today 35 (June 2008) 32-35.

H.H. Wiendahl, G. Von Cieminski and H.P. Wiendahl, Stumbling blocks of PPC: Towards the holistic configuration of PPC systems, Production Planning \& Control 16 (2005) 634-651. 